\title{
Hypoglycémie artéfactuelle chez une patiente atteinte d'anémie falciforme
}

\author{
Linda R. Wang MD, Justin Morein MD, Christopher McCudden PhD, Alexander Sorisky MDCM
}

- Citation : CMAJ 2021 November 1;193:E1660-2. doi : 10.1503/cmaj.210191-f

Voir la version anglaise de l'article ici : www.cmaj.ca/lookup/doi/10.1503/cmaj.210191

$\mathbf{U}$ ne femme de 53 ans a été dirigée vers notre clinique d'endocrinologie pour hypoglycémie récurrente détectée lors d'analyses sanguines. Ses valeurs de glycémie à jeun au cours de l'année écoulée variaient de 2,6 à 3,4 mmol/L. Même si elle était asymptomatique, on lui donnait du jus comme traitement chaque fois qu'un tel résultat était signalé.

La patiente souffrait d'anémie falciforme homozygote compliquée, de lupus érythémateux disséminé, de sclérose en plaques, de névrite optique et de dysfonction vésicale. Elle ne souffrait pas de diabète sucré et n'utilisait pas d'hypoglycémiants. Ses médicaments incluaient : prednisone $(5 \mathrm{mg} / \mathrm{j}$, dose stable depuis 2 ans), azathioprine, desmopressine, acide folique, vitamine D, vitamine B12, olmésartan, toltérodine, gabapentine et suppléments de calcium. Elle recevait une exsanguino-transfusion toutes les 8 semaines et ses taux d'hémoglobine $S$ avant les exsanguino-transfusions demeuraient toujours au-dessus de $50 \%$; à la demande de la patiente, depuis 1 an, la fréquence des exsanguino-transfusions avait été réduite, car auparavant, elles avaient lieu toutes les 4 semaines. Elle n'avait pas d'antécédents familiaux de néoplasie endocrinienne ou de diabète.

Étant donné que la patiente ne rapportait aucun symptôme d'hypoglycémie, nous avons soupçonné que les valeurs de glycémie basses puissent être fausses. Nous avons noté que les précédents spécimens de sang veineux avaient tous été prélevés avant l'exsanguino-transfusion en présence d'une réticulocytose massive (numération des réticulocytes pouvant atteindre

Tableau 1 : Valeurs de la glycémie chez une femme de 53 ans souffrant d'anémie falciforme, selon la méthode utilisée

\section{Méthode}

Résultats,

Échantillon de sang veineux sans inhibiteur de la glycolyse (plusieurs mesures)

$\mathrm{mmol} / \mathrm{L}$

Échantillon de sang capillaire (2 fois par jour pendant 2 semaines)

Échantillon de sang entier, à jeun, avec analyseur de gaz sanguins

Échantillon de sang veineux, à jeun, recueilli dans un tube renfermant du fluorure de sodium

\section{Points clés}

- Les patients qui présentent des taux nettement élevés de cellules sanguines nucléées, comme des leucocytes ou des réticulocytes, peuvent présenter une hypoglycémie artéfactuelle en raison d'une consommation excessive du glucose par ces cellules dans le tube de prélèvement avant l'analyse de laboratoire.

- Chez ces patients, on obtiendra une mesure plus précise avec une glycémie capillaire (au bout du doigt) et en analysant l'échantillon dans les 30 minutes, ou en utilisant des tubes qui contiennent un inhibiteur de la glycolyse.

- L'hypoglycémie vraie se traduit généralement par la triade de Whipple : taux faible de glucose sérique avec symptômes d'hypoglycémie au moment du prélèvement de l'échantillon montrant un taux de glucose bas et résolution des symptômes après un traitement approprié de l'hypoglycémie.

- En l'absence de symptômes d'hypoglycémie touchant le système nerveux autonome et symptômes neuroglycopéniques, des taux faibles de glucose sanguin devraient éveiller les soupçons à l'égard d'une hypoglycémie artéfactuelle, et il faut vérifier le glucose au moyen d'autres méthodes avant d'entreprendre des examens diagnostiques qui mobilisent beaucoup de ressources.

401,5 [plage normale 22-92] × 10\%/L). Cela pouvait suggérer une consommation excessive de glucose par les réticulocytes métaboliquement actifs dans le tube de prélèvement avant que le laboratoire ne procède à l'analyse.

Nous avons demandé à la patiente de mesurer sa glycémie capillaire 2 fois par jour pendant 2 semaines (à jeun et postprandiale) et ses valeurs de glycémie étaient normales, soit 3,9$5,1 \mathrm{mmol} / \mathrm{L}$. De plus, lorsque nous avons mesuré la glycémie sur du sang entier au moyen de l'analyseur de gaz sanguins (pour abréger l'intervalle entre le prélèvement et l'analyse) et sur un échantillon de sang recueilli dans un tube à prélèvement renfermant du fluorure de sodium/oxalate de potassium (inhibiteur de la glycolyse), nous avons obtenu des glycémies à jeun normales, de 4,6 et de 4,8 $\mathrm{mmol} / \mathrm{L}$, respectivement (tableau 1). Les spécimens prélevés avant la présente consultation donnaient des lectures hypoglycémiques et étaient recueillis dans des tubes sans inhibiteur de la glycolyse. Les 3 méthodes ont été utilisées en 
période de réticulocytose massive $\left(481,6 \times 10^{9} / \mathrm{L}\right.$ lors de la glycémie capillaire et $594,6 \times 10^{9} / \mathrm{L}$ lors du prélèvement sanguin [plage normale $\left.22-92 \times 10^{9} / \mathrm{L}\right]$ ).

Nous avons informé la patiente et ses médecins qu'elle ne souffrait pas d'hypoglycémie vraie et qu'elle n'avait pas besoin d'examens ou de suivis qui mobilisent beaucoup de ressources. S'il fallait procéder à des glycémies lorsque les taux de réticulocytes étaient élevés, nous avons suggéré d'utiliser l'une des méthodes décrites plus haut, la plus pratique étant la glycémie capillaire hors laboratoire.

\section{Discussion}

L'hypoglycémie vraie est exceptionnelle chez les patients qui ne souffrent pas de diabète sucré. Des examens plus approfondis sont nécessaires pour en distinguer certaines des causes rares, telles que : hyperinsulinisme endogène, tumeurs non liées aux cellules des îlots, insuffisance surrénalienne, maladie grave, septicémie, défaillance organique, grave malnutrition et effets indésirables de certains médicaments comme la gatifloxacine et l'indométhacine ${ }^{1}$. Toutefois, avant de demander ces tests, il faut avant tout déterminer si les mesures de glycémie basses correspondent bien à une hypoglycémie vraie.

\section{Triade de Whipple}

Un réel épisode hypoglycémique se définit par la triade de Whipple : signes ou symptômes d'hypoglycémie affectant notamment le système nerveux autonome et symptômes neuroglycopéniques, glycémie basse au moment des symptômes et résolution des symptômes après la correction des taux de glycémie ${ }^{1}$.

Les symptômes d'hypoglycémie affectant le système nerveux autonome incluent : tremblements, palpitations, diaphorèse, sensation de faim, anxiété et paresthésie. Les symptômes neuroglycopéniques incluent : faiblesse, confusion, changements comportementaux, altération du niveau de conscience et convulsions ${ }^{1}$.

\section{Hypoglycémie artéfactuelle}

Chez les patients exposés à un risque d'hypoglycémie (p. ex., diabète de longue date), une hypoglycémie asymptomatique devrait éveiller les soupçons à l'égard d'un phénomène artéfactuel. Il faut mesurer la glycémie au moyen d'une autre méthode avant de demander des examens plus approfondis.

En général, on se fie à la glycémie du sang veineux pour confirmer l'hypoglycémie avec un test capillaire hors laboratoire. Toutefois, ce cas illustre que la glycémie sur du sang veineux peut parfois donner des valeurs erronément basses. Chaque méthode peut comporter des erreurs qui lui sont propres (tableau 2) et connaître les limites de chacune permet aux médecins de choisir le test qui convient le mieux pour confirmer un résultat inhabituel.

Les erreurs préanalytiques sont importantes et pourtant ce sont des causes peu reconnues de glycémies veineuses faussement basses, la plus commune étant la glycolyse in vitro causée par des cellules métaboliquement actives, qui survient entre le prélèvement du spécimen et son analyse. L'Association américaine du diabète recommande de placer les échantillons sur de la glace et de séparer le plasma des cellules dans les 30 minutes pour réduire les risques de glycolyse ${ }^{10}$; mais cela n'est pas toujours possible en pratique. L'importance de ce phénomène a été mise en lumière par une étude australienne de 2020 qui a examiné le traitement des échantillons pour des tests oraux de tolérance au glucose (75 g) chez des femmes enceintes soumises au dépistage du diabète de grossesse; avec un protocole de centrifugation rapide, les taux de diabète de grossesse ont augmenté de $11,6 \%$ à $20,6 \%$, illustrant l'incidence importante des processus préanalytiques pour la santé publique ${ }^{11}$.

\section{Incidence des cellules métaboliquement actives}

Une quantité excessive de cellules nucléées peut amplifier la glycolyse in vitro et l'hypoglycémie artéfactuelle risque alors d'atteindre un niveau alarmant. L'hypoglycémie artéfactuelle causée par ce mécanisme s'observe en général chez des patients qui

Tableau 2 : Sources fréquentes d'artéfacts lors des contrôles glycémiques

\begin{tabular}{|c|c|c|}
\hline Méthode & Cause des valeurs de glycémie faussement basses & Cause des valeurs de glycémie faussement élevées \\
\hline Glycémie capillaire & $\begin{array}{l}\text { - Circulation diminuée }{ }^{2} \text { (hypotension, maladie de Raynaud, } \\
\text { maladie vasculaire périphérique) } \\
\text { - Erreurs d'analyse }{ }^{3} \text { (hématocrite élevé, taux d'oxygène élevés } \\
\text { [>100 mm Hg], bandelette-test périmée) } \\
\text { - Erreur du technicien }{ }^{3}\end{array}$ & $\begin{array}{l}\text { - Substances sucrées sur la peau } \\
\text { - Hématocrite bas }{ }^{3} \\
\text { - Solutions de dialyse péritonéale contenant de l'icodextrine }{ }^{3 *} \\
\text { - Taux élevés d'acide urique }{ }^{3 *} \\
\text { - Taux faibles d'oxygène }\end{array}$ \\
\hline Glucose veineux & $\begin{array}{l}\left.\text { - } \text { Leucocytose }^{1,2} \text { (leucémie }^{2} \text {, leucocytose réactive }{ }^{4}\right) \\
\text { - Réticulocytose } e^{1,2} \text { (polycythémie vraie }^{2,5} \text {, anémie hémolytique } \\
\left.\text { chronique }{ }^{6}\right) \\
\text { - Prolongation du délai de transport ou d'analyse }{ }^{1} \\
\text { - Hyperviscosité }\end{array}$ & \\
\hline $\begin{array}{l}\text { Surveillance du glucose } \\
\text { sous-cutané }\end{array}$ & $\begin{array}{l}\text { - } \text { Compression locale }^{7} \\
\text { - } \text { Présence d'acide salicylique }^{8} \\
\text { - } 12 \text { premières heures d'un nouveau senseur }\end{array}$ & $\begin{array}{l}\text { - Acétaminophène } e^{9 \star} \\
\text { - Hydroxyurée }{ }^{9} \\
\text { - Vitamine } C^{8}\end{array}$ \\
\hline
\end{tabular}


présentent une leucocytose extrême (p. ex., dans les cas de leucémie) $)^{2}$. Chez notre patiente, toutefois, la cause était probablement une réticulocytose massive causée par l'anémie falciforme. Ce phénomène a été peu rapporté comme cause de l'hypoglycémie artéfactuelle chez les patients qui présentent d'autres maladies telles que la polycythémie vraie et l'anémie hémolytique chronique ${ }^{5,12}$.

La prévalence de cet artéfact chez des patients souffrant d'anémie falciforme est difficile à estimer étant donné que la vérification de la glycémie n'est pas toujours indiquée en présence d'anémie falciforme. La glycémie a initialement été demandée par le néphrologue de notre patiente qui s'inquiétait d'une légère protéinurie et elle a ensuite été contrôlée à répétition en raison de la persistance des résultats anormaux.

La gravité de la réticulocytose sous-jacente influe sur la fréquence des contrôles pour cet arfétact. La gravité de l'anémie falciforme varie considérablement, tant selon le génotype qu'à l'intérieur de chacun. Notre patiente présentait une anémie falciforme à génotype ( $\mathrm{HbSS}$ ) et phénotype sévères et plusieurs complications. Ses hypoglycémies se sont manifestées après l'allongement de l'intervalle entre ses exsanguino-transfusions, passées de 4 à 8 semaines, entraînant des taux d'hémoglobine $\mathrm{S}$ de plus de $50 \%$ avant l'exsanguino-transfusion (cible < 30\%); et les mesures de glycémie basses étaient enregistrées avant les exsanguino-transfusions, au moment du pic de réticulocytose.

\section{Méthodes d'atténuation de la glycolyse excessive in vitro} En présence de taux nettement élevés de cellules nucléées, les méthodes les plus fiables de mesure de la glycémie sont celles qui abrègent l'intervalle entre le prélèvement et l'analyse comme ici, la glycémie capillaire hors laboratoire ou l'analyse de gaz sanguins veineux (effectuée dans les 5-10 min). Les tubes à prélèvement qui contiennent un inhibiteur de la glycolyse peuvent aussi donner des résultats plus précis en réduisant la consommation du glucose in vitro ${ }^{11,12}$. Les inhibiteurs de la glycolyse plus récents, tels que le citrate, sont associés à des résultats de glycémie plus précis que le fluorure, mais ne sont pas accessibles à grande échelle ${ }^{6}$.

Nous avons utilisé toutes les méthodes suggérées afin de confirmer que les faibles taux de glycémie veineuse initialement enregistrés chez notre patiente étaient artéfactuels, ce qui a permis d'éviter des tests plus approfondis superflus et qui mobilisent beaucoup de ressources. Chez un patient qui ne présente aucun symptôme d'hypoglycémie, avant d'entreprendre une batterie de tests approfondis indiqués pour confirmer une hypoglycémie vraie, il faut envisager l'hypothèse d'une hypoglycémie artéfactuelle.

\section{Références}

1. Cryer PE, Axelrod L, Grossman AB, et al.; Endocrine Society. Evaluation and management of adult hypoglycemic disorders: an Endocrine Society clinical practice guideline. J Clin Endocrinol Metab 2009;94:709-28.

2. Tarasova VD, Zena M, Rendell M. Artifactual hypoglycemia: an old term for a new classification. Diabetes Care 2014;37:e85-6.

3. Ginsberg BH. Factors affecting blood glucose monitoring: sources of errors in measurement. J Diabetes Sci Technol 2009;3:903-13.

4. Cryer P. Glucose homeostasis and hypoglycemia. In: Kronenberg H, Melmed S, Polonsky K, et al., editors. Williams textbook of endocrinology. 11th ed. Philadelphia: Saunders;2008:1503-33.

5. Macaron Cl, Kadri A, Macaron Z, et al. Nucleated red blood cells and artifactual hypoglycemia. Diabetes Care 1981;4:113-5.
6. Gambino R, Bruns DE. Stabilization of glucose in blood samples: out with the old, in with the new. Clin Chem Lab Med 2013;51:1883-5.

7. Mensh BD, Wisniewski NA, Neil BM, et al. Susceptibility of interstitial continuous glucose monitor performance to sleeping position. J Diabetes Sci Technol 2013;7:863-70.

8. Freestyle Libre 14 day indications and important safety information. Chicago: Abbott Laboratories. Accessible ici : https://provider.myfreestyle.com/safety -information.html (consulté le 30 mai 2021).

9. Interfering substances and risks. Dexcom. Accessible ici : https://www.dexcom. com/interference (consulté le 30 mai 2021).

10. Sacks DB, Arnold M, Bakris GL, et al.; National Academy of Clinical Biochemistry. Position statement executive summary: guidelines and recommendations for laboratory analysis in the diagnosis and management of diabetes mellitus. Diabetes Care 2011;34:1419-23.

11. Potter JM, Hickman PE, Oakman C, et al. Strict preanalytical oral glucose tolerance test blood sample handling is essential for diagnosing gestational diabetes mellitus. Diabetes Care 2020;43:1438-41.

12. Arem R, Jeang MK, Blevens TC, et al. Polycythemia rubra vera and artifactual hypoglycemia. Arch Intern Med 1982;142:2199-201.

\section{Intérêts concurrents : Aucun déclaré.}

Cet article a été révisé par des pairs.

Les auteurs ont obtenu le consentement de la patiente.

Affiliations : Division d'endocrinologie et de métabolisme, Service de médecine (Wang, Morein, Sorisky), Hôpital d'Ottawa et Département de médecine, Université d'Ottawa; Service de pathologie et de médecine de laboratoire (McCudden), Hôpital d'Ottawa et Département de pathologie et de médecine de laboratoire, Université d'Ottawa; Association des laboratoires régionaux de l'Est de l'Ontario (McCudden); Institut de recherche de l'Hôpital d'Ottawa (Sorisky), Hôpital d'Ottawa, Ottawa, Ont.

Collaborateurs : Linda Wang et Alexander Sorisky sont à l'origine de la conception du rapport. Tous les auteurs ont rédigé le manuscrit et en ont révisé de façon critique le contenu intellectuel important; ils ont donné leur approbation finale pour la version destinée à être publiée et assument l'entière responsabilité de tous les aspects du travail.

Propriété intellectuelle du contenu : Il s'agit d'un article en libre accès distribué conformément aux modalités de la licence Creative Commons Attribution (CC BY-NC-ND 4.0), qui autorise l'utilisation, la diffusion et la reproduction dans tout média à la condition que la publication originale soit adéquatement citée, que l'utilisation se fasse à des fins non commerciales (c.-à-d., recherche ou éducation) et qu'aucune modification ni adaptation n'y soit apportée. Voir : https://creativecommons.org/licenses/by-nc-nd/4.0/deed.fr.

Correspondance : Alexander Sorisky, asorisky@toh.ca

La section Études de cas présente de brefs rapports de cas à partir desquels des leçons claires et pratiques peuvent être tirées. Les rapports portant sur des cas typiques de problèmes importants, mais rares ou sur des cas atypiques importants de problèmes courants sont privilégiés. Chaque article commence par la présentation du cas (500 mots maximum), laquelle est suivie d'une discussion sur l'affection sous-jacente (1000 mots maximum). La soumission d'éléments visuels (p. ex., tableaux des diagnostics différentiels, des caractéristiques cliniques ou de la méthode diagnostique) est encouragée. Le consentement des patients doit impérativement être obtenu pour la publication de leur cas. Renseignements destinés aux auteurs : www.cmaj.ca. 